\title{
P01-81
}

\section{ASSOCIATION OF ADH2 AND ALDH2 GENE POLYMORPHISMS WITH ALCOHOL DEPENDENCE: A PILOT STUDY FROM INDIA}

P. Prasad, M. Vaswani

National Drug Dependence Treatment Center,Department of Psychiatry, All India Institute of Medical Sciences, Delhi, India

Background: Functional polymorphism in $\mathrm{ADH} 2$ and $\mathrm{ALDH} 2$ genes are considered most important among several genetic determinants of alcohol dependence (AD), a complex disorder.

Aims: There is no report on the widely studied Arg47His and Glu487Lys polymorphisms from Indian alcohol dependent population. We, for the first time, report allelic and genotypic frequencies of Arg47His and Glu487Lys SNPs in North Indian alcohol dependent subjects.

Methods: A total of $n=174$ alcohol dependent males, recruited using DSM IV criteria, were genotyped using PCR-RFLP method.

Results: Data obtained from genetic analysis was correlated with clinical parameters using T test or Mann Whitney's U test. ADH2 gene polymorphism was found to be largely monomorphic with minor allele frequency (ADH2*2) $<0.001$. For the ALDH2 Glu487Lys SNP, genotypic frequencies were $0.73\left(2^{*} 1 /{ }^{*} 1\right), 0.16\left(2^{*} 1{ }^{*} 2\right)$ and $0.11\left(2^{*} 2 /{ }^{*} 2\right)$, with minor allele frequency $\left(\operatorname{ALDH} 2^{*} 2\right)=0.19$. Various clinical parameters were found to be significantly associated with ALDH2 polymorphism.

Conclusions: The highlight of the study is a clear association of ALDH2 gene polymorphism with delayed onset and shorter duration of alcohol consumption among $A L D H 2 * 2 / 22$ individuals. Our finding bolsters the protection conferring property of the ALDH2*2 allele of Glu487Lys SNP of ALDH2 gene. 